\title{
Model-based supervision of valves in a flotation process
}

\author{
Michael Bask \\ Control Engineering Group \\ Luleå University of Technology \\ S-971 87 Luleå, SWEDEN \\ email:Michael.Bask@sm.luth.se
}

\author{
Andreas Johansson \\ Control Engineering Group \\ Luleå University of Technology \\ S-971 87 Luleå, SWEDEN \\ email:Andreas.Johansson@sm.luth.se
}

\begin{abstract}
An observer based approach for detecting clogging in valves in flotation processes is investigated. Integral action and linear feedback applied to a nonlinear process model constitutes the observer for which local stability is shown. The integral terms give estimates of the clogging in the valves of the process and this estimate is compared to a constant threshold. Experiments on real data from Boliden's flotation series at the Boliden Area Concentrator, Sweden, show no false alarm during any of the two working conditions, PI control and LQ control. It is also shown that cloggings, simulated by manipulating measurement data, are promptly detected. The observer based fault detection algorithm is compared to an algorithm based on parameter estimation and advantages of the two approaches are highlighted.
\end{abstract}

\section{INTRODUCTION}

Froth flotation is an important and versatile mineralprocessing technique in which the differences in physicochemical surface properties of particles from different minerals is used. By using flotation reagents it is possible to separate the valuable minerals from the rocky material called gangue. In reverse flotation the gangue is separated into the froth but in direct flotation the minerals are transferred to the froth. An introduction to flotation can be found in [1].

In both reversed and direct flotation it is important to control the levels of the flotation tanks, see [2] for an account on a multivariable LQ-controller for a flotation plant. The levels are controlled with valves on the outflow of each tank and to ensure that the controller is able to fulfill its performance requirements, a fault detection algorithm is needed to detect clogging of these valves in an early stage. Surveys of fault detection algorithms can be found in [3], [4] and for nonlinear systems in particular in [5], [6]. State observers are commonly used and examples of state observers for nonlinear systems are the Extended Kalman Filter (EKF), [7], [8], the Constant Gain EKF, [9] and the Extended Luenberger observer, [10]. A survey of nonlinear state observers can be found in [11] and a table comparing different nonlinear observer approaches in [12].

In [13], a two step algorithm for estimating valve parameters in a flotation plant is developed which can be used for detecting clogging. In the first step, an observer estimates the flow through the valves. The second step consist of a recursive estimation algorithm that uses the estimated flow from the first step to estimate a linear valve model. Drawbacks of this approach are that analysis regarding stability and robustness is not straightforward and that the valve model is restricted to be affine in the unknown parameters.
In this article we present an observer based one step algorithm to detect the clogging. The algorithm uses a nonlinear model of the process extended with integral action and corrected with a linear feedback term. The integral terms are shown to be estimates of the clogging.

Furthermore, an experimental comparison between the observer based algorithm and the parameter estimation technique, in [13], with respect to the clogging estimation is presented. See [14] for a theoretical comparison between observer based and parameter identification fault detection approaches for linear time invariant systems.

The article is composed as follows. First, a description of the flotation process model is presented in section II followed by an identification algorithm of the valve parameters in section III. The next section (IV), treats the residual generation and is divided into two parts. In the first part, a short description of the valve parameter estimation technique in [13] is given and in the second part, an observer estimating the clogging is introduced. In section $\mathrm{V}$, calculations and experimental results for a real flotation process is presented. Finally some conclusions are drawn in section VI.

\section{FLOTATION PROCESS MODEL}

The flotation process consists of cascade coupled tanks with control valves after each tank for the purpose of controlling the levels. The measured level in tank $i$ is denoted $h_{i}(t)$ where $i=1 . . n$ and $n$ is the number of tanks. The input signals to the process is the valve control signals $u_{i}(t)$ and the external inflows to the tanks $q_{i n, i}(t)$. The levels are measured in meter and the inflow in $m^{3} / h$. Figure 1 shows, as an example, a flotation process with four tanks.

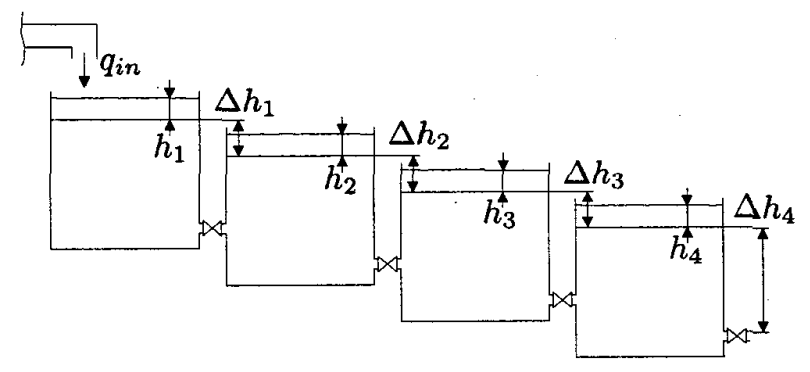

Fig. 1. The flotation process

The continuous time model of the levels $h(t)$ in the tanks can be described as a system of first order differential 
equations.

$$
\dot{h}(t)=F\left(h(t), u(t), \Delta C_{v}(t)\right)+B q_{i n}(t)
$$

where $h(t) \in \mathrm{R}^{n}, \Delta C_{v}(t) \in \mathrm{R}^{n}, u(t) \in \mathrm{R}^{n}, q_{\text {in }}(t) \in \mathrm{R}^{m}$ and $B \in \mathrm{R}^{n \times m}$ are, respectively, the state, clogging area, valve control signal, inflow and input matrix. The time argument $t$ is droped in the sequel to enhance readability. The vector of nonlinear functions $F\left(h, u, \Delta C_{v}\right)$ is

$$
F\left(h, u, \Delta C_{v}\right)=\left[\begin{array}{c}
f_{1}\left(h, u, \Delta C_{v}\right) \\
\vdots \\
f_{n}\left(h, u, \Delta C_{v}\right)
\end{array}\right]
$$

where

$$
f_{1}\left(h, u, \Delta C_{v}\right)=-\frac{\left(C_{v .1}\left(u_{1}\right)+\Delta C_{u .1}\right)}{\alpha_{1}} \sqrt{H_{1}(h)}
$$

and

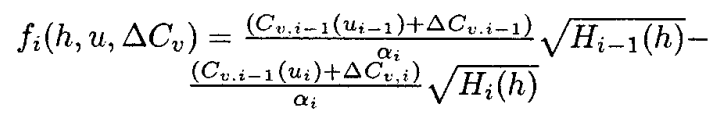

for $i \geq 2$. The function $C_{v, i}\left(u_{i}\right)$ gives the valve opening area of valve $i$, in $\mathrm{m}^{2}$, and $\alpha_{i}$ is the liquid surface area of tank $i$, also in $\mathrm{m}^{2}$, assuming it is a constant independent of the level in the tank. The functions $H_{i}(h)$ are defined as

$$
H_{i}(h)=2 g\left(h_{i}-h_{i+1}+\Delta h_{i}\right)
$$

where $g$ is the acceleration of gravity and $\Delta h_{i}$ is the physical height difference between the zero-levels of tank $i$ and $i+1$. For the last tank $H_{n}(h)=2 g\left(h_{n}+\Delta h_{n}\right)$ where $\Delta h_{n}$ is the height difference between the zero-level and the outflow valve. A reasonable assumption is that the clogging in the valves develops slowly and therefore $\Delta C_{v}(t)=0$. A process model for the extended state vector $x=\left[\begin{array}{ll}h & \Delta C_{v}\end{array}\right]^{T}$ can then be written as

$$
\begin{aligned}
\dot{x} & =\bar{F}(x, u)+\bar{B} q_{i n} \\
h & =\bar{C} x
\end{aligned}
$$

where $\bar{F}(x, u) \in \mathrm{R}^{2 n}$ and $\bar{B} \in \mathrm{R}^{2 n}$ such that

$\bar{F}(x, u)=\left[\begin{array}{c}F\left(h, u, \Delta C_{v}\right) \\ 0\end{array}\right], \bar{B}=\left[\begin{array}{c}B \\ 0\end{array}\right], \bar{C}=\left[\begin{array}{ll}I & 0\end{array}\right]$

\section{VALVE MODEL IDENTIFICATION}

The manufacturer of the valves suggests the following nonlinear model of the valve opening

$$
C_{v}(u)=a_{1} d^{2} \frac{a_{2} u+a_{3} u^{2}}{1+e^{a_{4} u}}
$$

where $a_{1}, \cdots, a_{4}$ are constants and $d$ is the diameter of the valve. The control signal $u$ is in the interval $0 \leq u \leq 1$ where $u=0$ and $u=1$ represents closed and open, respectively. A common and often sufficient approximation of the nonlinear valve model is the linear function

$$
C_{v}(u)=K u+c
$$

which is used in e.g. [13].

Simulations with the measurement data that are used in the experiments in section $\mathrm{V}$ have shown that the model (2) used with constants $a_{1}, \cdots a_{4}$ provided by the manufacturer does not work well so the linearized model (3) is used instead. The valve parameters $K$ and $c$ have to be identified which can be done by using the Least-square method. Assuming no clogging, the flow through a valve $i$ can be expressed as

$$
q_{i}(t)=\left(K_{i} u_{i}(t)+c_{i}\right) \sqrt{H_{i}(h(t))}
$$

From the process model (1) it follows that

$$
q_{i n}(t)-\alpha_{1} \dot{h}_{1}(t)=q_{1}(t)
$$

which, integrated from time $t_{0}$ to time $t_{1}$, yields

$$
\begin{gathered}
\int_{t_{0}}^{t_{1}} q_{i n}(\tau) d \tau-\alpha_{1}\left(h_{1}\left(t_{1}\right)-h_{1}\left(t_{0}\right)=\int_{t_{0}}^{t_{1}} q_{1}(\tau) d \tau=\right. \\
\int_{t_{0}}^{t_{1}}\left(K_{1} u_{1}(\tau)+c_{1}\right) \sqrt{H_{1}(h(\tau))} d \tau= \\
{\left[\begin{array}{cc}
\int_{t_{0}}^{t_{1}} u_{1}(\tau) \sqrt{H_{1}(h(\tau))} d \tau & \int_{t_{0}}^{t_{1}} \sqrt{H_{1}(h(\tau))} d \tau
\end{array}\right]\left[\begin{array}{c}
K_{1} \\
c_{1}
\end{array}\right]}
\end{gathered}
$$

The constants $K_{1}$ and $c_{1}$ can thus be estimated by the leastsquares method since $q_{i n}, h$ and $u$ are measured. Similar calculations can be done for the remaining valves utilizing the fact that the inflow to tank $i$ is the outflow from tank $i-1$.

\section{RESIDUAL GENERATION}

The fault detection task is to achieve an automatic supervision system for clogging in the control valves. The problem consists of designing an observer to estimate the amount of clogging and construct thresholds that indicate when there is too much clogging in a valve.

Fault detection algorithms can generally be divided into two parts. First, a state observer or a parameter estimation algorithm is utilized to generate a residual which is supposed to be nonzero in case of fault and zero otherwise. Then, a threshold is employed to take a decision if a fault is present.

In the sequel, a parameter estimation algorithm for clogging detection [13] is briefly recapitulated in section IV-A and a state-observer based approach is introduced in section IV-B. The estimates of the clogging area is used as residual and it is compared to a constant threshold.

\section{A. A short summary of the parameter estimation algorithm from [13]}

The algorithm presented in [13] is a residual generator consisting of two steps. First, observer based estimates of the flows through the valves are generated. Analysis shows that these flow estimates are low-pass filtered versions of the actual flows.

Second, estimates $\hat{K}_{i}$ and $\hat{c}_{i}$ of the parameters of the valve model $q_{i}=K_{i} u_{i}+c_{i}$ are generated by using a recursive directional forgetting algorithm, [15]. For more details see [13]. 
An estimate of the clogging area can be calculated as the difference between the valve opening obtained from the online estimated parameters $\hat{K}_{i}$ and $\hat{c}_{i}$ and the valve opening calculated by using parameters estimated off-line with e.g. the algorithm in section III.

$$
\hat{\Delta C}_{v, i, p e}=\hat{K}_{i} u_{i}(t)+\hat{c}_{i}-\left(K_{i} u_{i}(t)-c_{i}\right)
$$

which can be directly compared to the estimate $\Delta C_{v}$, generated by the observer introduced in the next section.

\section{B. Observer}

The suggested observer structure for estimating the clogging $\Delta C_{v}$ of the valves is

$$
\begin{aligned}
& \dot{\hat{h}}=F\left(\hat{h}, u, \Delta C_{v}\right)+B q_{i n}+L_{1}(h-\hat{h}) \\
& \dot{\Delta C_{v}}=L_{2}(h-\hat{h})
\end{aligned}
$$

where $L_{1}, L_{2} \in \mathrm{R}^{n \times n}$ are the observer gain matrices. The observer can also be written as

$$
\begin{aligned}
& \dot{\hat{x}}=\bar{F}(\hat{x}, u)+\bar{B} q_{i n}+\bar{L}(h-\hat{h}) \\
& \hat{h}=\bar{C} \hat{x}
\end{aligned}
$$

where $\bar{L}=\left[\begin{array}{ll}L_{1} & L_{2}\end{array}\right]^{T}$.

Other observer structures have been considered, such as the EKF, [7], [8] and the Luenberger like observer [16]. However, as will be shown later, the linear feedback term is sufficient to stabilize the estimation error system since $\frac{\partial \bar{F}(x, u)}{\partial x}$ does not vary too much. An advantage with choosing the feedback term linear is that Thau's theorem [17] can be utilized to show stability of the observer. Different algorithms have been suggested of how to design the feedback gain $\bar{L}$. In [9] a method based on the EKF is suggested and other approaches are described in [18] and [19].

A definition of the Lipschitz condition is needed for the stability analysis of the observer.

Definition 1: Assume the function $\Phi(x, u)$ is continuous in $x$ and there exist a finite constant $\gamma$ such that for all $x_{1}, x_{2} \in \mathrm{X}$ and $u \in \mathrm{U}$ the inequality

$$
\left\|\Phi\left(x_{1}, u\right)-\Phi\left(x_{2}, u\right)\right\| \leq \gamma\left\|x_{1}-x_{2}\right\|
$$

holds where $\|\cdot\|$ denotes the euclidian vector norm. Then $\Phi$ is said to be locally Lipschitz and $\gamma$ its lipschitz constant. If $\mathrm{X}=\mathrm{R}^{\mathrm{n}}$ then it is said that the function $\Phi$ is globally Lipschitz.

A condition established by Thau [17] can be used to investigate the stability of the observer (4).

Theorem 1: (Thau [17]): Assume that the process is described by

$$
\begin{aligned}
& \dot{x}=A x+G\left(u, q_{i n}\right)+\Phi(x, u) \\
& h=C x
\end{aligned}
$$

and the observer is

$$
\dot{\hat{x}}=A \hat{x}+G\left(u, q_{i n}\right)+\Phi(\hat{x}, u)+L(h-C \hat{x})
$$

If $(A, C)$ is detectable and

$$
\gamma<\frac{\lambda_{\min }(Q)}{2 \lambda_{\max }(P)}
$$

where the matrices $P$ and $Q$ are symmetric and positive definite and satisfy the Lyapunov equation

$$
(A-L C)^{T} P+P(A-L C)=Q
$$

then

$$
\lim _{t \rightarrow \infty}(x(t)-\hat{x}(t))=0
$$

It has been shown in [20] that the ratio in (7) can be maximized by choosing the matrix $Q=I$.

To show stability by using Thau's theorem, the process has to be rewritten in the form of (5). In order to obtain a small value for the Lipschitz constant $\gamma, \Phi(x, u)$ is chosen as the higher order terms of a Taylor expansion of $\bar{F}(x, u)$.

$$
\begin{gathered}
\bar{F}(x, u)=\bar{F}\left(x_{0}, u_{0}\right)+\bar{F}_{x}\left(x_{0}, u_{0}\right)\left(x-x_{0}\right)+ \\
+\bar{F}_{u}\left(x_{0}, u_{0}\right)\left(u-u_{0}\right)+\Phi(x, u)
\end{gathered}
$$

where $\bar{F}_{x}=\frac{\partial \bar{F}}{\partial x}$ and $\bar{F}_{u}=\frac{\partial \bar{F}}{\partial u}$. By defining $A=\bar{F}_{x}\left(x_{0}, u_{0}\right)$ and $\beta=\bar{F}_{u}\left(x_{0}, u_{0}\right), \Phi(x, u)$ and $G\left(u, q_{i n}\right)$ can be expressed as

$$
\begin{gathered}
\Phi(x, u)=\bar{F}(x, u)-\bar{F}\left(x_{0}, u_{0}\right)-A\left(x-x_{0}\right)-\beta\left(u-u_{0}\right) \\
G\left(u, q_{i n}\right)=\bar{F}\left(x_{0}, u_{0}\right)-A x_{0}+\beta\left(u-u_{0}\right)+\bar{B} q_{i n}
\end{gathered}
$$

From the inequality formulation of the mean value theorem [21] it follows that if the function $\Phi$ is continuous and differentiable on the set $\mathrm{D}$ and has bounded partial derivatives, then $\gamma$ is the 2-norm of the jacobian matrix of $\Phi(x, u)$.

The function $\bar{F}(x, u)$ is continuous and differentiable and thus also $\Phi(x, u)$ since

$$
\frac{\partial \Phi(x, u)}{\partial x}=\bar{F}_{x}(x, u)-\frac{\partial}{\partial x}(A x)=\bar{F}_{x}(x, u)-A
$$

If inequality (7) is fulfilled then the observer is asymptotically stable and $\hat{x} \rightarrow x$ as $t \rightarrow \infty$ which implies that

$$
\widehat{\Delta C_{v}} \rightarrow \Delta C_{v}
$$

\section{EXPERIMENTAL RESULTS}

Experiments have been carried out on data from Boliden's flotation series at the Boliden Area Concentrator, Sweden. This is a direct flotation process and consists of four tanks i.e. $n=4$ and the only inflow is to tank one. Levels and valve control signals for all four tanks and the inflow are measured.

The flow $q_{i n}$ into the process is around $200 \mathrm{~m}^{3} / \mathrm{h}$ but is varying severely between $50 \mathrm{~m}^{3} / \mathrm{h}-350 \mathrm{~m}^{3} / \mathrm{h}$.

Identification of the valve parameters using the presented algorithm from section III resulted in

$$
\begin{aligned}
K & =\left[\begin{array}{llll}
5.566 & 4.158 & 5.918 & 5.716
\end{array}\right]^{T} 10^{-4} \\
c & =\left[\begin{array}{llll}
30.02 & 61.59 & 35.85 & -11.66
\end{array}\right]^{T} 10^{-4}
\end{aligned}
$$

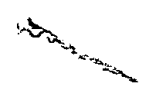


By experiments, it has been found that suitable values for the feedback gain are

$$
L=\left[\begin{array}{cccc}
6 & 0 & 0 & 0 \\
0 & 6 & 0 & 0 \\
0 & 0 & 6 & 0 \\
0 & 0 & 0 & 6 \\
0.04 & 0 & 0 & 0 \\
0 & 0.04 & 0 & 0 \\
0 & 0 & 0.04 & 0 \\
0 & 0 & 0 & 0.04
\end{array}\right]
$$

To check for stability of the observer, the matrix $A$ in (6) is expressed as the jacobian matrix of the function $\bar{F}(x, u)$ at the equilibrium point $x=x_{0}=\left[\begin{array}{ll}h_{0} & \Delta C_{v, 0}\end{array}\right]^{T}, u=$ $u_{0}$ and $q_{i n}=q_{i n, 0}$. The equilibrium values of the control signals $u_{0}$ are found by choosing $x_{0}$ and $q_{i n, 0}$ and solving the equation

$$
\bar{F}\left(x_{0}, u_{0}\right)+B q_{i n, 0}=0
$$

which is obtained by setting $\dot{x}=0$ in the process model. The chosen equilibrium point and some physical parameters are

$$
\begin{aligned}
h_{0} & =\left[\begin{array}{llll}
0.4 & 0.4 & 0.4 & 0.4
\end{array}\right]^{T} \\
\Delta C_{v, 0} & =\left[\begin{array}{llll}
0 & 0 & 0 & 0
\end{array}\right]^{T} \\
q_{i n, 0} & =200 \\
\alpha & =\left[\begin{array}{llll}
17 & 25 & 17 & 17
\end{array}\right]^{T} \\
\Delta h & =\left[\begin{array}{llll}
0.4 & 0.4 & 0.8 & 1.5
\end{array}\right]^{T}
\end{aligned}
$$

Choosing $Q=I, L=\bar{L}, C=\bar{C}$ and $A=\bar{F}_{x}\left(x_{0}, u_{0}\right)$, it is straightforward to calculate the quotient in (7) and obtain $\frac{\lambda_{\min }(Q)}{2 \lambda_{\max }(P)}=0.0398$.

In order to calculate the lipschitz constant $\gamma$ some reasonable assumptions on the state $x$ must be made. Specifically, it is assumed that the levels will not vary more than \pm 0.1 $m$ and that the clogging will not be larger then $50 \%$ of the maximum valve opening. If the valve control signal is allowed to vary between

$$
0 \leq u(t) \leq 1
$$

then the lipschitz constant is $\gamma=0.0287$ and since the inequality (7) is thus fulfilled, the observer is shown to be locally stable. With the assumption that the level variations are $\pm 0.1 \mathrm{~m}$, the clogging can be allowed to be as much as $98 \%$ before the stability limit, $\gamma=0.0398$ is reached. If the clogging is allowed to be $50 \%$, the levels can be allowed to vary $\pm 0.225 \mathrm{~m}$ while maintaining stability of the observer.

Two different working conditions exist, either the flotation process is controlled by a multivariable LQ-controller, described in [2], or by four separate PI-controllers. When the PI-controllers are used, the levels and the control signals vary extensively. An example of this can be seen in Figure 2 , which shows the level measurement in tank 2 for a time
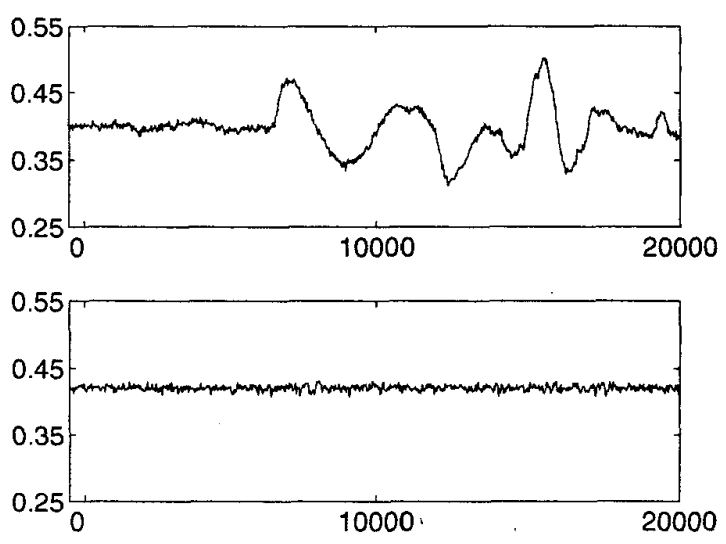

Fig. 2. The level measurement of tank 2 when using PI control (upper picture) and LQ control (lower picture).

interval with PI-controller and another time interval when the LQ-controller is operating.

The amount of clogging in a valve in a real process can not be measured in practice, so to investigate if the presented algorithm produces a satisfying result, a clogging has to be simulated. A clogging means that the actual valve opening is less than expected and can thus be simulated by adding a positive quantity to the control signal which gives total control of the fault. A reasonable assumption is that when clogging occurs it is a relatively slow process and then the valve control signal will increase as almost the only evidence of a clogging. In our experiment, a ramp has been added to the control signal of valve 2 . The ramp rises 0.10 units starting at $t=4000$ seconds and finishes at 14000 seconds thus imitating an increasing clogging in the valve. The modified and the unchanged control signal of valve two is shown in Figure 3 for an interval with the PI-controller and one with the LQ-controller in operation.

The clogging estimate from the observer in section IV-B is compared to a constant threshold which is ten percent of the fully open valve area. There are more advanced methods then a constant threshold, for example statistical methods [22] and deterministic methods [4], but this is to be a topic of future development of the clogging detection algorithm. Both working conditions are evaluated, Figure 4 shows the clogging estimation when the PI-controllers are used and Figure 6 when the LQ-controller is in operation. It can be noted that there is no false alarm in either of the conditions since no clogging estimate except for the clogged second valve exceeds the threshold. However, with the PI-controller, the estimates are closer to the limits so there is an imminent risk of false alarms.

An interesting comparison is when using the observer based algorithm presented in section IV-B and the parameter estimation algorithm, [13] described in section IV-A. None of the algorithms are optimized so a performance comparison 

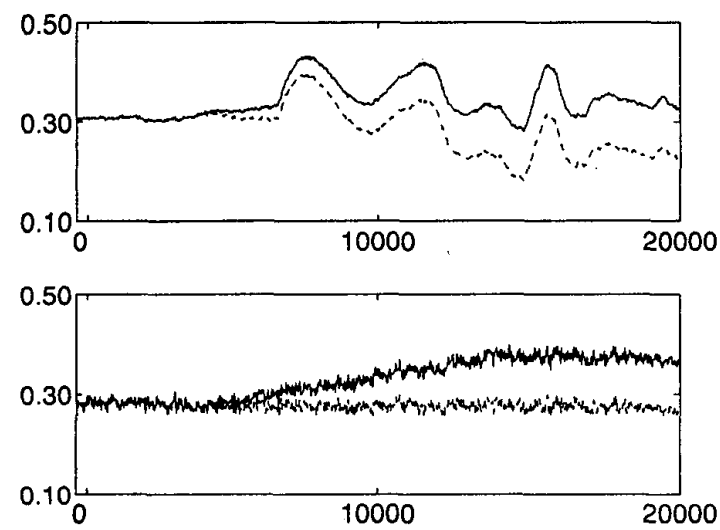

Fig. 3. The control signal of valve 2 is shown when using PI control (upper picture) and LQ control (lower picture). Each picture shows the modified (solid) and unmodified (dashed) control signal.

is not relevant. What is more interesting is to see that the results from the two algorithms seem to be quite similar. The final values of the estimated clogging areas seem to be fairly similar and even the behavior, compare Figure 4 to Figure 5 and Figure 6 to Figure 7. It can also be noted that the recursive algorithm seems to be more sensitive to disturbances and produces false alarms in valve 3 and 4 , but this may be caused by poor tuning of the algorithm. Finally, it can be concluded that a robust threshold needs to be derived in order to decrease the risk for false alarms, independent of which residual generator that is used.

An advantage with the parameter estimation algorithm is that estimates of the valve parameters are obtained on-line which can provide information on other faults in the valve.

For the observer based approach two advantages may be pointed out. First, concerning the clogging estimation, the results are similar to the parameter estimation algorithm but the algorithm is more straightforward to analyze. Therefore, it is also very likely that designing a robust thresholds is easier. Second, changing to a more complex valve model, e.g. the nonlinear function (2), is simple.

\section{CONCLUSION}

An observer based algorithm to estimate the amount of clogging in the valves of flotation processes is proposed and evaluated. This algorithm is also compared to a parameter estimation algorithm presented in [13].

In the observer based approach, the observer consists of a nonlinear process model extended with integral action and corrected by a linear feedback term. Local stability of this observer is shown. The integral terms give estimates of the clogging which are compared to constant thresholds.

Experiments have been carried out with measurement data from Boliden's flotation series at the Boliden Area Concentrator, Sweden. The flotation process operates under

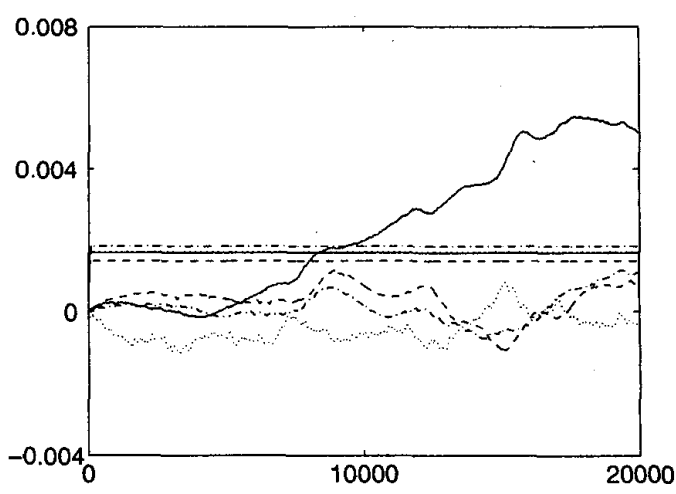

Fig. 4. The observer based clogging estimates (section IV-B) and thresholds (straight lines) are shown when PI controllers are in operation and with simulated clogging in valve 2 . Clogging estimate for valve 1 (dotted), valve 2 (solid), valve 3 (dash-dotted) and valve 4 (dashed).

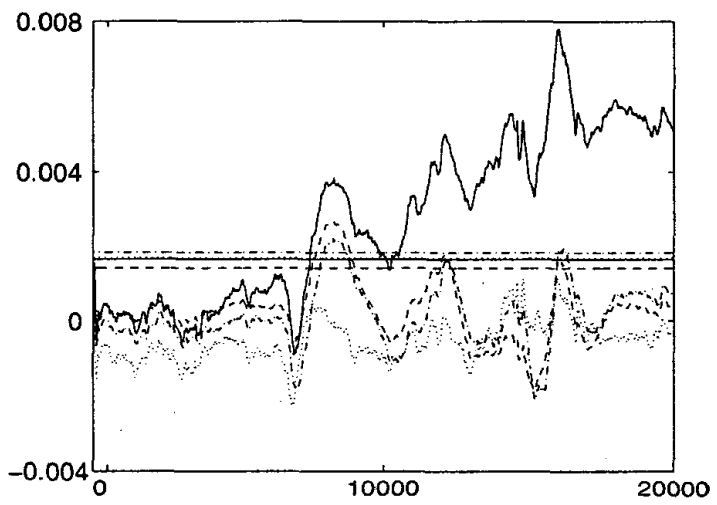

Fig. 5. This shows the same situation as in Figure 4 above but using the parameter estimation based algorithm from [13] instead.

two different working conditions, either four separate PIcontrollers or a multivariable LQ-controller is used. The fault detection algorithms are shown to be effective for both working conditions but the risk of false alarms is higher when the PI-controllers are used since there are more variations in the levels in this case.

The observer based algorithm is compared to a parameter estimation approach and both algorithms have similar problems regarding disturbances. Therefore, it is desirable to derive a robust threshold for evaluating the clogging estimate.

\section{ACKNOWLEDGEMENT}

The authors would like to thank Urban Olofsson at Boliden for providing measurements from the flotation series at Boliden Area Concentrator. Financial support by Vinnova of the project AIS33 is gratefully acknowledged. 


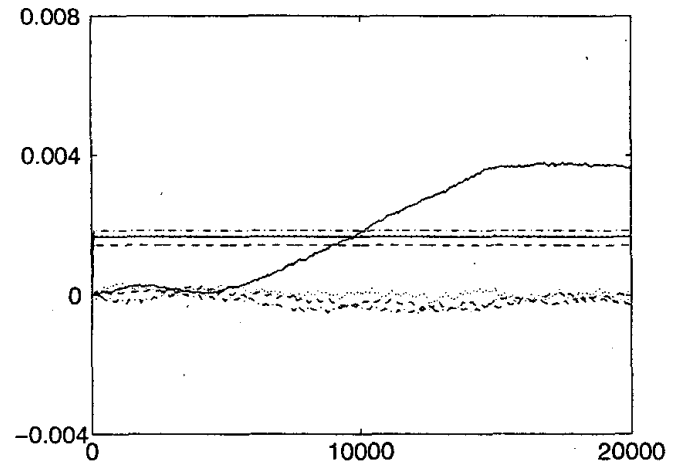

Fig. 6. The observer based clogging estimates (section IV-B) and thresholds (straight lines) are shown when LQ controllers are in operation and with simulated clogging in valve 2 . Clogging estimate for valve 1 (dotted), valve 2 (solid), valve 3 (dash-dotted) and valve 4 (dashed).

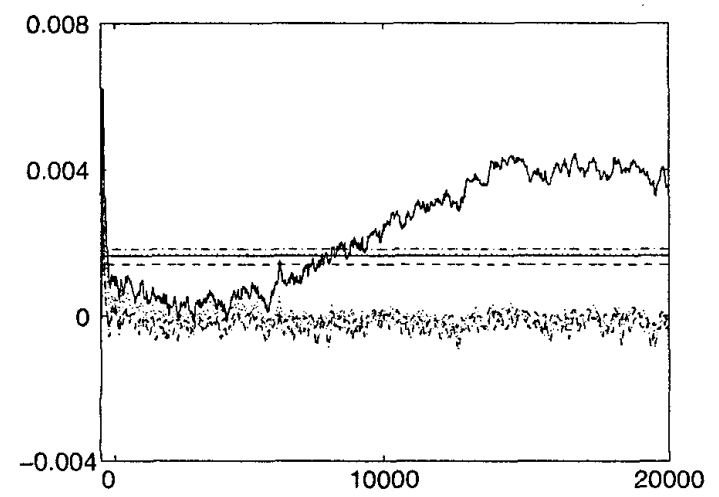

Fig. 7. This shows the same situation as in Figure 6 above but using the parameter estimation approach from [13] instead.

\section{REFERENCES}

[1] B.A. Wills, Mineral processing technology, Butterworth-Heinemann, 1997.

[2] B. Stenlund and A. Medvedev, "Level control of cascade coupled flotation tanks", Control engineering practice, vol. 10, no. 4, pp. 443-448, 2002.

[3] P. M. Frank, "Fault diagnosis in dynamic systems using analytical and knowledge-based redundancy - a survey and some new results", Automatica, vol. 26, no. 3, pp. 459-474, 1990.

[4] P. M. Frank, "Survey of robust residual generation and evaluation in observer-based fault detection systems", Journal of Process Control, vol. 7, no. 6, pp. 403-424, 1997.

[5] E. Alcorta García and P. M. Frank, "Deterministic nonlinear observer-based approaches to fault diagnosis: A survey", Control engineering practice, vol. 5, no. 5, pp. 663-670, 1997.
[6] V. Krishnaswami and G. Rizzoni, "A survey of observer based residual for FDI", in IFAC symposium on Fault Detection, Supervision and Safety for Technical Processes- Safeprocess '94, 1994, pp. 34-39.

[7] A. Gelb, Applied Optimal Estimation, MIT Press: Cambridge, MA, 1974.

[8] A. H. Jazwinski, Stochastic Processes and Filtering Theory, Academic Press: New York, 1970.

[9] M. G. Safonov and M. Athans, "Robustness and computational aspects of nonlinear stochastic estimators and regulators", IEEE Transactions on Automatic Control, vol. 23 , no. 4 , pp. $717-725,1978$.

[10] S. Valluri and M. Soroush, "Nonlinear state estimation in the presence of multiple steady 'states", Industrial and Engineering Chemistry Research, vol. 35, no. 8, pp. 2645-2659, 1996.

[11] E. A. Misawa and J. K. Hedrick, "Nonlinear observers a state-of-the-art survey", Journal of Dynamic Systems, Measurement and Control, vol. 111, no. 3, pp. 344-352, 1989.

[12] G Wang, S Peng, and H Huang, "A sliding observer for nonlinear process control", Chemical Engineering Science, vol. 52, no. 5, pp. 787-805, 1997.

[13] B. Stenlund and A. Medvedev, "Supervision of control valves in a series of cascade coupled flotation tanks", in 4th IFAC workshop on on-line fault detection and supervision in the chemical process industries, 2001.

[14] E. Alcorta García and P. M. Frank, "On the relationship between observer and parameter identification based approaches to fault detection", in 13th triennial world congress, 1996, pp. 25-29.

[15] L. Cao and H. Schwartz, "A directional forgetting algorithm based on the decomposition of the information matrix", Automatica, vol. 36, no. 11, pp. 1725-1731, 2000.

[16] G. Ciccarella, M. Dalla Mora, and A. Germani, "A luenberger-like observer for nonlinear systems", International Journal of Control, vol. 57, no. 3, pp. 537-556, 1993.

[17] F.E. Thau, "Observing the state of nonlinear dynamic systems", International Joumal of Control, vol. 17, no. 3, pp. 471-479, 1973.

[18] R. Rajamani, "Observers for lipschitz nonlinear systems", IEEE Transactions on Automatic Control, vol. 43, no. 3, pp. 397-401, 1998.

[19] S. Raghavan and J.K. Hedrick, "Observers design for a class of nonlinear systems", International Journal of Control, vol. 59, no. 2, pp. 515-528, 1994.

[20] R.V. Patel and M.Toda, "Quantitative measures of robustness in multivariable systems", 1980.

[21] J. Dieudonné, Foundations of modern analysis, Academic press, New York and London, 1969.

[22] M. Basseville and I. V. Nikiforov, Detection of Abrupt Changes, Prentice-Hall, 1993. 